bioRxiv preprint doi: https://doi.org/10.1101/2020.04.20.050468; this version posted April 20, 2020. The copyright holder for this preprint (which was not certified by peer review) is the author/funder, who has granted bioRxiv a license to display the preprint in perpetuity. It is made available under aCC-BY-NC-ND 4.0 International license.

\title{
NMR backbone assignment and dynamics of Profilin from Heimdallarchaeota
}

\author{
Syed Razaul Haq ${ }^{1}$, Sabeen Survery ${ }^{1}$, Fredrik Hurtig ${ }^{1}$, Ann-Christin Lindås ${ }^{1}$ and Celestine N. Chi ${ }^{2}$. \\ ${ }^{1}$ Department of Molecular Bioscience, Wenner-Gren Institute, Stockholm University, Svante Arrhenius v. 20C, SE- \\ 10691 Stockholm, Sweden \\ ${ }^{2}$ Department of Medical Biochemistry and Microbiology, Uppsala University, BMC Box 582, SE-75123 Uppsala, \\ Sweden.
}

*Corresponding authors: ann.christin.lindas@su.se, chi.celestine@imbim.uu.se

Keywords: profilin, Archaea, chemical shifts, eukaryogenesis, actin, NMR spectroscopy 


\section{Abstract}

The origin of the eukaryotic cell is an unsettled scientific question. The Asgard superphylum has emerged as a compelling target for studying eukaryogenesis due to the previously unseen diversity of eukaryotic signature proteins. However, our knowledge about these proteins is still relegated to metagenomic data and very little is known about their structural properties. Additionally, it is still unclear if these proteins are functionally homologous to their eukaryotic counterparts. Here, we expressed, purified and structurally characterized profilin from Heimdallarchaeota in the Asgard superphylum. The structural analysis shows that while this profilin possess similar secondary structural elements as eukaryotic profilin, it contains additional secondary structural elements that could be critical for its function and an indication of divergent evolution.

\section{Biological context}

The origin of the eukaryotic cell remains an unsettled scientific question and several hypotheses have been put forward to explain the complex evolutionary history of the eukaryotic cell ${ }^{1234}$. The Woese hypothesis proposes three domains of life - Archaea, Bacteria and Eukaryota, with independent evolutionary trajectories ${ }^{5}$. The eocyte hypothesis suggests the existence of only two domains - bacteria and archaea, and that eukaryotes emerged from the symbiotic relationship of an unknown archaeal host with an alphaproteobacterium ${ }^{6}$. Recently, environmental metagenomic sampling led to the discovery of the Asgard superphylum. Comparative genomic analysis of Asgard archaea and eukaryotes appears to support the eocyte hypothesis ${ }^{7}$. The genomes of Asgardarchaea are enriched with proteins previously considered eukaryote-specific, so called eukaryotic signature proteins (ESPs), and phylogenetic analysis placed the Asgardarchaea in a monophyletic group with eukaryotes ${ }^{8}$.

Actin plays a crucial part of the eukaryotic cytoskeleton and is essential to many processes, including cellular motility, cell division, endocytosis, intracellular cargo transport, amongst many other ${ }^{9}$. Because of the central role actin plays in the eukaryotic cell, the sequence of actin remains highly conserved among eukaryotes. While actin homologues are widespread throughout all domains of life, the dynamic actin cytoskeleton and the regulatory actin-binding proteins are a hallmark of eukaryotic life.

The Asgard genomes contain close actin homologues and several actin-binding proteins; including profilin, gelsolin, Arp $2 / 3$ complex subunit 4 and a large family of small GTPases that regulate the actin cytoskeleton in eukarotes $^{8}$. This posits the question; do these archaea possess an actin cytoskeleton with complex regulation analogous with the eukaryotic cytoskeleton? While metagenomic analysis has identified these proteins, their 
21 which makes in vivo comparison with eukaryotic homologs difficult ${ }^{10}$. Currently, protein production in heterologous expression systems and reconstitution of the purified complexes in vitro represents one of the best approaches in characterizing their function. Profilin is expressed in most, if not all, eukaryotic cells and is one of the most important proteins in regulating actin cytoskeletal dynamics ${ }^{11}$. Eukaryotic profilin (eprofilin) is a small protein (approximately 14-19 kDa) which sequester monomeric G-actin from the cytoplasmic pool, thus controlling polymerization $^{12}$. Despite significant divergence at the sequence level, the eprofilin tertiary structure is wellconserved and folds into 3D structures constituting $7 \quad \beta$-strands and $4 \alpha$-helices ${ }^{13}$. eprofilin promotes the elongation of actin filament assembly at the barbed end by acting as a nucleotide exchange factor, and by interacting with elongation factors such as Ena/Vasp, Formins, and Wasp ${ }^{14-16}$. These nucleation factors bind eprofilin through a polyproline motif at a domain physically separate from the actin binding-site. Moreover, eprofilin can also bind to phosphatidylinositol 4,5-bisphosphate $\left(\mathrm{PIP}_{2}\right)^{17}$ at the plasma membrane which results in a reduced affinity towards polyproline and actin $^{17}$. eprofilin also competes with phospholipase $C$ for PIP $_{2}$ binding which leads to interference with the PI3K/AKT signaling pathway ${ }^{18}$.

Recently, it has been shown that profilins encoded in several lineages of the Asgardarchaea not only share structural similarity with eukaryotic orthologues but are able to regulate the function of eukaryotic actin. This implies that profilin from Asgardarchaea have the potential of complex regulation of the hypothetical actin cytoskeleton as well ${ }^{19}$. In contrast to human profilin I, a previous study showed that the Asgard profilins (Loki 1 and 2, Thor, Odin and Heimdall) did not show polyproline binding. This led the authors to suggest that Asgard profilins do not bind polyproline, and that polyproline directed actin assembly is a later addition in eukaryotic evolution ${ }^{19}$. However, $\mathrm{PIP}_{2}$ was shown to modulate the affinity of Asgard profilin towards rabbit actin in a functional assay ${ }^{19}$. Nevertheless, some of the Asgard genomes are incomplete and the structural and functional relationships of representative profilins from different Asgard lineages are still poorly understood. It might therefore be too early to assume that Asgard profilins do not bind polyproline. In addition, the crystal structures of various profilins combined with functional data do not only reveal structural similarity between Asgard profilins, but also highlights some subtle differences at the species level ${ }^{19}$. Within the Asgard superphylum, the Heimdallarchaeota appears to currently be the closest relative of eukaryotes ${ }^{7}$. Here we present the NMR backbone assignment and dynamics of the Heimdallarchaeota profilin (heimProfilin) as a first step towards characterizing it structurally. These NMR amino acid specific assignments and dynamics provide for the first time an atomic snapshot of heimProfilin as well as providing further evidence for the idea that the Asgard encoded proteins possess similar structural elements and are likely to perform similar roles as those in eukaryotes.

\section{Methods and experiments}

\section{Protein expression and purification}

Heimdallarchaeota profilin (GenBank: OLS22855.1) was cloned into the pSUMO-YHRC vector, kindly provided by Claes Andréasson (Addgene Plasmid \#54336; RRID: Addgene_54336) with an N-terminal 6xHistidine-tag and a SUMO-tag (cleavable with Ulp1 protease). The vector was transformed and expressed in E. coli Rosetta DE3 cells. Initially, the cells were grown in $2 x \mathrm{TY}$ media at $37^{\circ} \mathrm{C}$ until the optical density of the culture was 0.8 at $600 \mathrm{~nm}$. 
58 The cells were then transferred into $\mathrm{M} 9$ media supplemented with $1 \mathrm{~g} / \mathrm{L}{ }^{15} \mathrm{~N}$-ammonium chloride and $1 \mathrm{~g} / \mathrm{L}{ }^{13} \mathrm{C}$ -

59 glucose and grown for 1 hour at $30^{\circ} \mathrm{C}$. Protein expression was induced by $0.5 \mathrm{mM}$ IPTG. For Deuterium $\left({ }^{2} \mathrm{H}\right)$

60 labelling, the $\mathrm{M} 9$ medium was prepared with $100 \%$ or $50 \% \mathrm{D}_{2} \mathrm{O}$ and cells were grown overnight at $30{ }^{\circ} \mathrm{C}$. Post-

61 induction, the cells were harvested by centrifugation and resuspended in the binding buffer $(50 \mathrm{mM}$ Tris- $\mathrm{HCl} \mathrm{pH}$

$627.5,0.3 \mathrm{M} \mathrm{NaCl}, 1 \mathrm{mM}$ TCEP, $10 \mathrm{mM}$ imidazole, 10\% glycerol). The cells were then lysed by sonication and the cell

63 Iysate was clarified by centrifugation at $25,000 \times \mathrm{g}$ for $45 \mathrm{~min}$ at $4{ }^{\circ} \mathrm{C}$ and finally filtered through a $0.2 \mu \mathrm{M}$ syringe

64 filter (Sarstedt). The supernatant was loaded onto a His GraviTrap column (1ml, GE healthcare) and the bound

65 protein was eluted with binding buffer containing $250 \mathrm{mM}$ imidazole. The protein was incubated with Ulp1

66 protease overnight at $4{ }^{\circ} \mathrm{C}$ to cleave the SUMO-tag including the Histidine-tag. The protein was desalted using a

67 PD10 column (GE Healthcare) and loaded onto a His GraviTrap column again to remove the tag and the Ulp1

68 protease. The protein was concentrated using a 10,000 NMWL cutoff centrifugal filter (Merck Millipore) and

69 further purified on a Superdex 75 10/300 GL (GE Healthcare) size exclusion column, equilibrated with 25 mM Tris-

$70 \mathrm{HCl}, 50 \mathrm{mM} \mathrm{NaCl}, 5 \%$ Glycerol, $1 \mathrm{mM}$ TCEP at pH 7.5. Protein concentration was determined using the molar 71 absorption coefficient at $280 \mathrm{~nm}(29450 / \mathrm{M} / \mathrm{cm})$.

\section{NMR Spectroscopy}

Double labeled ${ }^{15} \mathrm{~N},{ }^{13} \mathrm{C}$, or triple labeled ${ }^{15} \mathrm{~N},{ }^{13} \mathrm{C},{ }^{2} \mathrm{H}$ were prepared to a concentration of $20 \mathrm{mg} / \mathrm{ml}$ in $25 \mathrm{mM}$ Tris$\mathrm{HCl}, 50 \mathrm{mM} \mathrm{NaCl}$, 5\% Glycerol, $1 \mathrm{mM}$ TCEP at pH 7.5 and thereafter supplemented with $3 \% \mathrm{D}_{2} \mathrm{O}$ and $0.03 \%$ sodium azide. The NMR assignment experiments were performed at $308 \mathrm{~K}$ on a triple-resonance Bruker 900, 700 or 600 $\mathrm{MHz}$ spectrometers equipped with a cryogenic probe. NMR relaxation experiments were performed on a $600 \mathrm{MHz}$ spectrometer at $298 \mathrm{~K}$. Backbone sequence-specific assignments were carried out using the following experiments 2D ${ }^{1} \mathrm{H}^{15} \mathrm{~N}$-TROSY, 3D TROSY-HNCACCB, 3D TROSY-HNCA, 3D TROSY-CO) CACB and 3D TROSY-HN(CO)CA. For sidechain assignments, 2D ${ }^{1} \mathrm{H}-{ }^{13} \mathrm{C} C \mathrm{CT}-\mathrm{HSQC}, 3 \mathrm{D} \mathrm{HBHA}(\mathrm{CO}) \mathrm{NH}$ and 3D HCCH-TOCSY spectra were utilized. For assignment and fold verification 3D NOESY as well as ${ }^{3} J_{\mathrm{HNH}}$ for secondary structure verification were measured. For Backbone $R_{1}, R_{2}$ rates and hetero-nuclear NOES were determined in an interleaved manner with the experiments from the Bruker pulse program library. For $R_{1}$ and $R_{2}$ rates, the relaxation delay was sampled for 9 and 8 delay-durations which were pseudo-randomized, respectively $\left(R_{1}: 20,60,100,200,400,600,800\right.$ and $1000 \mathrm{~ms}$ and $R_{2}: 16,33,67$, $136,170,203,237$ and $271 \mathrm{~ms})$. The relaxation delay time was up to $1.5 \mathrm{~s}$ for $R_{1}$ and $1 \mathrm{~s}$ for $R_{2}$. The $\left[{ }^{1} \mathrm{H}\right]{ }^{15} \mathrm{~N}$-hetNOE experiment and a reference spectra were recorded with a total $2 \mathrm{~s}^{1} \mathrm{H}$ saturation time for the NOE experiment and the same recovery time for the reference experiment. The order parameter $S^{2}$ and the internal correlation time were calculated with the program dynamic center. The rotational diffusion tensor was estimated from the ratio of the relaxation rates $\left(R_{1}\right.$ and $\left.R_{2}\right)$. TALOS and CYANA were employed to predict secondary structure, using ${ }^{1} \mathrm{H}^{\mathrm{N}},{ }^{15} \mathrm{~N}$, and ${ }^{13} \mathrm{C}^{\alpha}$ chemical shifts. All other data were processed with topspin and analyzed using CCPNMR ${ }^{20}$ and $C Y A N A^{21}$.

\section{Assignment and data deposition}

The expressed and purified heimProfilin corresponds to the full length as was generated from metagenomics data ${ }^{8}$. acids (see methods). This profilin possesses a 20 -amino acid extension compared with the previously characterized 

eprofilins or those from Loki I and II and Odin. We obtained up to $88 \%$ of all backbone and up to $80 \%$ of all sidechain assignments. 135 of the 148 non-proline amide residues were assigned in the ${ }^{1} \mathrm{H}-{ }^{15} \mathrm{~N}$ TROSY (Fig. 1). The following amides were not possible to assign: M1, K2, D3, 16, K11, K14, 119, S25, E27, N62, S85 and N89. The missing amides could be due to motional broadening or fast solvent exchange. We obtained $92 \%$ of the $C_{\alpha}$ and $C_{\beta}$ resonance assignments. $H_{\beta}$ and $H_{\alpha}$ proton shifts were completed to $97 \%$ and $96 \%$, respectively. These assignments were further verified by ${ }^{15} \mathrm{~N} /{ }^{13} \mathrm{C}$ 3D NOESY spectra. Backbone and side-chain chemical shifts assignments have been deposited to the Biological Magnetic Resonance Data Bank (BMRB) with the Accession Number 50190.

\section{Secondary structure analysis}

The structures of eukaryotic and Asgard profilin from Loki (1 and 2) and Odin have been determined by X-ray crystallography ${ }^{19}$. However, no structural information is available from the heimProfilin which appears to be the closest relative to the eukaryotes. With the completed assignments, it was now possible to analyze the secondary structure characteristics of this profilin to see if it adopts similar secondary structural elements. Analysis of sequential and medium range NOEs revealed stretches of $d N N, d N N(i, i+2), d \alpha \beta(i, i+3), d \alpha N(i, i+3)$. Residues 29$33,64-68,124-127$ and 125-144 continual revealed daN $(i, i+4)$ NOEs, indicating the presence of helices in this region. This is supported by the ${ }^{3} J_{\mathrm{HNH} \alpha}$ coupling constants for these residues which display small values typical of alpha helices (Fig. 2). ${ }^{13} C_{\alpha}$ and $C_{\beta}$ shifts are frequently used to predict secondary structure propensities. $C_{\alpha}$ shifts generally tend to shift upfield in a beta-sheet and extended strands relative to the random coil values. In alpha helices, these $C_{\alpha}$ shifts tend to shift downfield ${ }^{22}$. For $C_{\beta}$ values the opposite is true, they shift downfield for betasheets and extended strands and upfield for alpha helices. The $C_{\alpha}$ and $C_{\beta}$ values relative to random coil values are shown in figure 2. Examination of these plots indicates clear helical regions covering residues $29-34,64-68,124-$ 127 and 135-144. The helical region between residues $64-68$ has not been observed in previous profilin structures. The region of beta strands also agrees with NOEs values and slightly increased ${ }^{3}{ }_{\mathrm{HNH \alpha}}$ values. This analysis indicates that the overall secondary structural elements are preserved from archaea to eukaryotes albeit with some slight differences in their lengths. In addition, we observed an additional helix between residues 64-68 which was not present in the previously determined profilin structures. This might be important for modulating profilin-actin interaction and other physiological roles.

\section{Backbone dynamics}

$R_{1}$ and $R_{2}$ rates in addition to $\left[{ }^{1} \mathrm{H}\right]-{ }^{15} \mathrm{~N}$ hetNOE are frequently used to estimate the flexibility of proteins $\mathrm{s}^{23}$. Deviation of $R_{1}$ and $R_{2}$ rates for $\left[{ }^{1} \mathrm{H}\right]{ }^{15} \mathrm{~N}$ moieties from the average value often indicate a change in motional property. $R_{1}$ values that are larger than the average indicates the presence of flexibility in the ps-ns time range. On the other hand, $R_{2}$ rates with higher values than the average indicates regions of slow conformational exchange in the $\mu s-\mathrm{ms}$ time scale. $\left[{ }^{1} \mathrm{H}\right]-{ }^{15} \mathrm{~N}$ hetNOE with negative or near zero values indicate regions of high flexibility with motions faster than approximately $1 \mathrm{~ns}$. We measured and plotted the longitudinal $R_{1}$ and transverse $R_{2}$ rates as well the $\left[{ }^{1} \mathrm{H}\right]-{ }^{15} \mathrm{~N}$ hetNOE versus amino acids sequence (Fig. 3). Overall, the results from these values indicate a highly rigid protein between residues 25-148 (Fig. 2). However, N-terminal residues 1-24 show a high degree of flexibility, which is 
bioRxiv preprint doi: https://doi.org/10.1101/2020.04.20.050468; this version posted April 20, 2020. The copyright holder for this preprint (which was not certified by peer review) is the author/funder, who has granted bioRxiv a license to display the preprint in perpetuity. It is made available under aCC-BY-NC-ND 4.0 International license.

130 reflected in the very low $\left[{ }^{1} \mathrm{H}\right]-{ }^{15} \mathrm{~N}$ hetNOE values (Fig. 2). We also back calculate order parameter $S^{2}$ and internal

131 correlation time te. A plot of the calculated order parameter $S^{2}$ and internal correlation time te is shown in figure

$1323 \mathrm{~d}$. As shown in the plot, only the $\mathrm{N}$-terminal 1-24 amino acids show some degree of flexibility with very low order

133 parameter and high degree of internal motion. A few residues along the protein sequence indicate some degree of

134 flexibility. We determined the correlation time tc of $11.3 \mathrm{~ns}$. This value is slightly higher for a protein of this size

135 indicating probably due to the extended $\mathrm{N}$-terminal loop not completely structured.

\section{Conclusions}

138 In this study, we have determined the NMR backbone and dynamic data of a profilin from Heimdallarchaeota in 139 the Asgard superphylum. Our secondary structure analysis indicates that this profilin possess similar structural 140 elements to eukaryotic homologues, all beit at varied lengths. Our data also indicates that the heimProfilin appears 141 rigid apart from $\mathrm{N}$-terminal residues 1-24 which are not present in previously characterized eukaryotic profilins. 142 We observed an additional helix between residue 64-68 which lies in the interface of the actin binding site when 143 compared to eukaryotic profilin, and likely plays a role in modulating acting polymerization.

\section{Acknowledgments:}

\section{Declarations}

Funding: This work was supported by Wenner-Gren Stiftelsen Fellow's Grants, Ake Wiberg, Magnus Bergvall and O.E Edla Johannsson foundation grants to CC, Swedish Research Council Grant 621-2013-4685 for FH and Wellcome Trust Grant 203276/F/16/Z for SRH, SS and FH. This study made use of the NMR Uppsala infrastructure, which is funded by the Department of Chemistry - BMC and the Disciplinary Domain of Medicine and Pharmacy. Conflicts of interest/Competing interests: the authors declare no conflict of interest. Ethics approval: not applicable. Consent to participate: not applicable. Consent for publication: all authors read an approved the manuscript. Availability of data and material: all data and material are available and can be obtain from the authors

Figure 1 | $1 \mathrm{H}-15 \mathrm{~N}$ TROSY correlation spectrum of Heimdallarchaeota profilin. All ${ }^{1} \mathrm{H}-{ }^{15} \mathrm{~N}$ pairs that were assigned in this study. Side-chains of Glutamine and Asparagine are not assigned or shown.

Figure 2 | Secondary structure characterization of Heimdallarchaeota profilin. (a) Sequence-specific ${ }^{13} \mathrm{C}_{\alpha}$ secondary chemical shifts $\left(\delta \Delta^{13} C_{\alpha}\right)$ along the amino acid sequence of heimProfilin. (b) ${ }^{3} J_{H_{N H \alpha}}$ couplings plotted as a function of amino acid sequence. (c) TALOS secondary structure prediction based on ${ }^{1} \mathrm{H},{ }^{15} \mathrm{~N}$ and ${ }^{13} \mathrm{C}_{\alpha}$ shifts plotted as function of amino acid sequence. All three suggest the presence of helical and extended strands in similar regions. The presence of helices between residues 29-34, 64-68, 124-127 and 135-144 are clearly visible. ${ }^{3} \mathrm{H}_{\mathrm{HNH} \alpha}$ couplings are generally lower for helices $(2-4 \mathrm{~Hz})$ and higher for beta strands and extended regions $(2-8 \mathrm{~Hz})$. Very few ${ }^{3} J_{\text {HNH } \alpha}$ 
bioRxiv preprint doi: https://doi.org/10.1101/2020.04.20.050468; this version posted April 20, 2020. The copyright holder for this preprint (which was not certified by peer review) is the author/funder, who has granted bioRxiv a license to display the preprint in perpetuity. It is made available under aCC-BY-NC-ND 4.0 International license.

couplings were obtained for residues $1-20$. However, the ${ }^{13} \mathrm{C}_{\alpha}$ shifts and the TALOS prediction clearly shows that this region is extended.

Figure 3 | Dynamic characterization of the backbone based on $\left[{ }^{1} \mathrm{H}-\right]^{15} \mathrm{~N}$. (a) Longitudinal ${ }^{15} \mathrm{~N} R_{1}$ relaxation rates plotted as a function of amino acid sequence. (b) Transverse ${ }^{15} \mathrm{~N} R_{2}$ relaxation rates versus the amino acid sequence. (c) $\left[{ }^{1} \mathrm{H}\right]^{15} \mathrm{~N}$-hetero-nuclear NOE data (hetNOE) along the amino acid sequence. (d) calculated $S^{2}$ order parameter (left axis) and internal motion $\tau$ (right axis) plotted as a function of the amino acid sequence. All parameters indicate a very rigid molecule structure from the $\mathrm{N}$-terminal 20 amino acids which show some degree of ps-ns motion based on the elevated $R_{1}$, lower hetNOE and lower $\mathrm{S}^{2}$ order parameter.

Figure 1

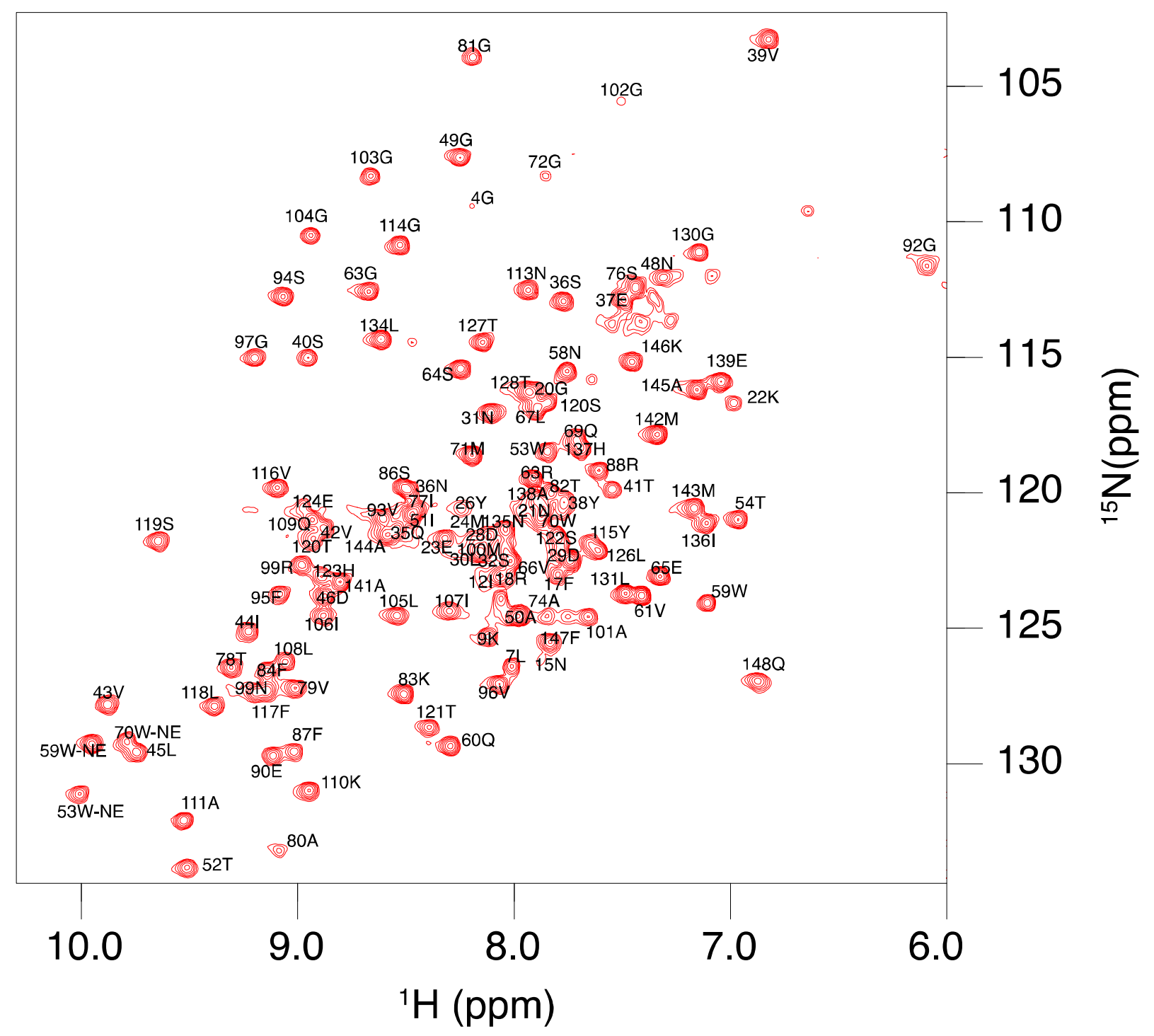


bioRxiv preprint doi: https://doi.org/10.1101/2020.04.20.050468; this version posted April 20, 2020. The copyright holder for this preprint (which was not certified by peer review) is the author/funder, who has granted bioRxiv a license to display the preprint in perpetuity. It is made available under aCC-BY-NC-ND 4.0 International license.

Figure 2

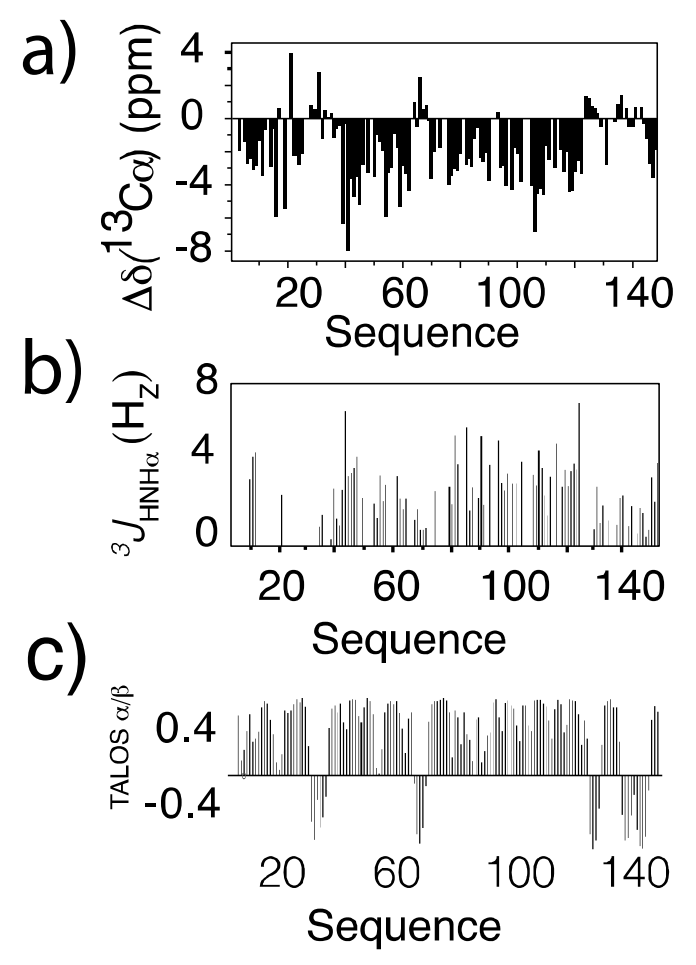

Figure 3 
a)
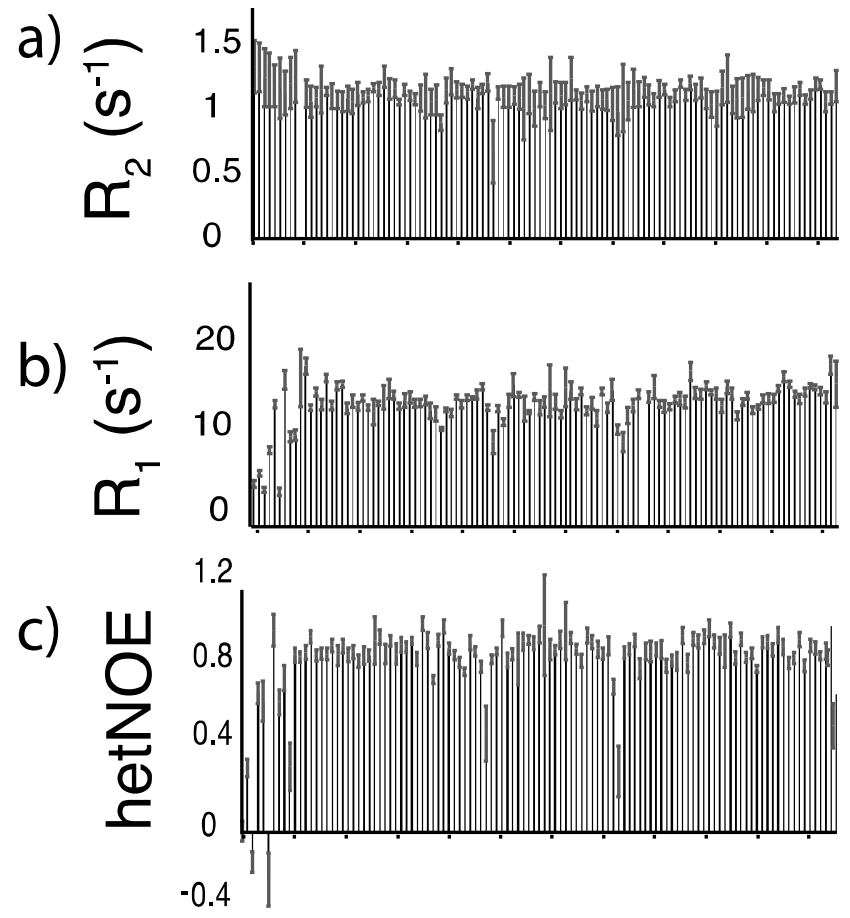

d)

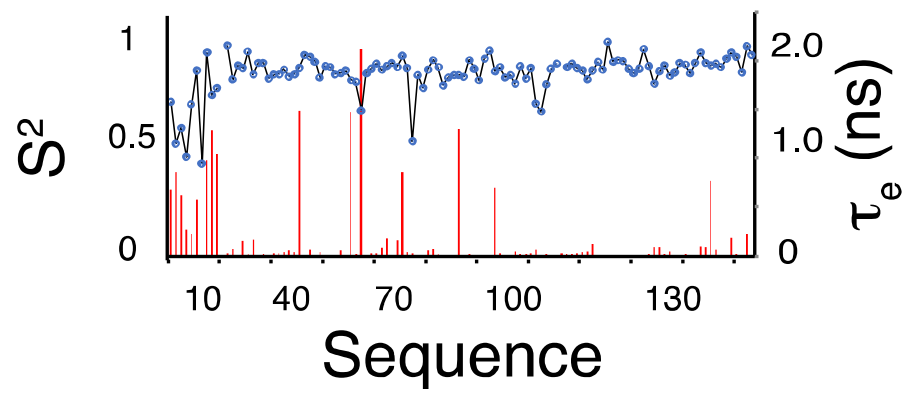

References:

1. Koonin, E.V. Origin of eukaryotes from within archaea, archaeal eukaryome and bursts of gene gain: eukaryogenesis just made easier? Philos Trans R Soc Lond B Biol Sci 370, 20140333 (2015).

2. $\quad$ Lopez-Garcia, P. \& Moreira, D. Open Questions on the Origin of Eukaryotes. Trends Ecol Evol 30, 697708 (2015).

3. Da Cunha, V., Gaia, M., Gadelle, D., Nasir, A. \& Forterre, P. Lokiarchaea are close relatives of Euryarchaeota, not bridging the gap between prokaryotes and eukaryotes. PLoS Genet 13, e1006810 (2017).

4. Spang, A. et al. Asgard archaea are the closest prokaryotic relatives of eukaryotes. PLoS Genet 14, e1007080 (2018).

5. Woese, C.R., Kandler, O. \& Wheelis, M.L. Towards a natural system of organisms: proposal for the domains Archaea, Bacteria, and Eucarya. Proc Natl Acad Sci U S A 87, 4576-9 (1990).

6. Lake, J.A., Henderson, E., Oakes, M. \& Clark, M.W. Eocytes: a new ribosome structure indicates a kingdom with a close relationship to eukaryotes. Proc Natl Acad Sci U S A 81, 3786-90 (1984).

7. Spang, A. et al. Complex archaea that bridge the gap between prokaryotes and eukaryotes. Nature 521, 173-179 (2015).

8. Zaremba-Niedzwiedzka, K. et al. Asgard archaea illuminate the origin of eukaryotic cellular complexity. Nature 541, 353-358 (2017).

9. Pollard, T.D. Actin and Actin-Binding Proteins. Cold Spring Harb Perspect Biol 8(2016).

10. Imachi, H. et al. Isolation of an archaeon at the prokaryote-eukaryote interface. Nature 577, 519-525 (2020).

11. Schluter, K., Jockusch, B.M. \& Rothkegel, M. Profilins as regulators of actin dynamics. Biochim Biophys Acta 1359, 97-109 (1997).

12. Birbach, A. Profilin, a multi-modal regulator of neuronal plasticity. Bioessays 30, 994-1002 (2008).

13. Schutt, C.E., Myslik, J.C., Rozycki, M.D., Goonesekere, N.C. \& Lindberg, U. The structure of crystalline profilin-beta-actin. Nature 365, 810-6 (1993). 
173 14. Reinhard, M. et al. The proline-rich focal adhesion and microfilament protein VASP is a ligand for profilins. EMBO J 14, 1583-9 (1995).

15. Kursula, P. et al. High-resolution structural analysis of mammalian profilin 2 a complex formation with two
physiological ligands: the formin homology 1 domain of mDia1 and the proline-rich domain of VASP. $J$ Mol Biol 375, 270-90 (2008).

16. Goldschmidt-Clermont, P.J. et al. The control of actin nucleotide exchange by thymosin beta 4 and profilin. A potential regulatory mechanism for actin polymerization in cells. Mol Biol Cell 3, 1015-24 (1992).

17. Lambrechts, A. et al. The mammalian profilin isoforms display complementary affinities for PIP2 and proline-rich sequences. EMBO J 16, 484-94 (1997).

18. Sathish, K. et al. Phosphorylation of profilin regulates its interaction with actin and poly (L-proline). Cell Signal 16, 589-96 (2004).

19. Akil, C. \& Robinson, R.C. Genomes of Asgard archaea encode profilins that regulate actin. Nature 562, 439-443 (2018).

186

187

20. Vranken, W.F. et al. The CCPN data model for NMR spectroscopy: development of a software pipeline. Proteins 59, 687-96 (2005).

21. Guntert, P., Mumenthaler, C. \& Wuthrich, K. Torsion angle dynamics for NMR structure calculation with the new program DYANA. J Mol Biol 273, 283-98 (1997).

190 22. Berjanskii, M.V. \& Wishart, D.S. A simple method to predict protein flexibility using secondary chemical shifts. J Am Chem Soc 127, 14970-1 (2005).

192

23. Palmer, A.G. Enzyme Dynamics from NMR Spectroscopy. Accounts of Chemical Research 48, $457-465$ (2015). 